Kragujevac Journal of Mathematics

Volume 41(1) (2017), Pages 105-120.

\title{
ON GENERALIZED DERIVATION IN RINGS AND BANACH ALGEBRAS
}

\author{
M. A. RAZA ${ }^{1}$ AND N. U. REHMAN ${ }^{2}$
}

\begin{abstract}
Let $R$ be a prime ring, $F$ be a generalized derivation associated with a derivation $d$ of $R$ and $m, n$ be the fixed positive integers. In this paper we study the case when one of the following holds: (i) $F(x) \circ_{m} F(y)=(x \circ y)^{n}$, (ii) $F(x) \circ{ }_{m} d(y)=d(x \circ y)^{n}$ for all $x, y$ in some appropriate subset of $R$. We also examine the case where $R$ is a semiprime ring. Finally, as an application we obtain some range inclusion results of continuous or spectrally bounded generalized derivations on non-commutative Banach algebras.
\end{abstract}

\section{INTRODUCTION}

In 1994, Bell and Daif [5] initiated the study of strong commutativity-preserving (scp) maps and proved that a nonzero right ideal $I$ of a semiprime ring is central, if $R$ admits a derivation which is scp on $I$. In 2002, Ashraf and Rehman [3] discussed the action of derivation in prime ring. More precisely they showed that if $R$ is a 2-torsion free prime ring, $I$ is a nonzero ideal of $R$ and $d$ is a nonzero derivation of $R$ such that $d(x) \circ d(y)=x \circ y$ for all $x, y \in I$, then $R$ is commutative. In 2007, Huang [17] proved that if $R$ is a prime ring with $\operatorname{char}(R) \neq 2, U$ is a square closed Lie ideal of $R$ and $F$ is a generalized derivation associated with derivation $d$ of $R$ such that $d(x) \circ F(y)=x \circ y$ for all $x, y \in U$, then either $d=0$ or $R$ is commutative. In [7], Bell and Rehman consider the similar situation where the derivation replaced by generalized derivation. More specifically, they studied $F(x) \circ F(y)=x \circ y$. In 2012, Huang [18] proved that if $R$ is a prime ring, $I$ a nonzero ideal of $R, n$ a fixed positive integer and $F$ a generalized derivation associated with a nonzero derivation

\footnotetext{
Key words and phrases. Prime and semiprime rings, generalized derivation, generalized polynomial identity (GPI), ideal.

2010 Mathematics Subject Classification. Primary: 16W25. Secondary: 16N60, 16 U80.

Received: February 19, 2016.
}

Accepted: August 14, 2016. 
$d$ such that $(F(x \circ y))^{n}=x \circ y$ for all $x, y \in I$, then $R$ is commutative. Many authors in this direction studied several identities in which various techniques are developed to investigate the conditions under which a ring becomes commutative or almost commutative. For more details and references, see $[1,2,6,7,13,15,16,18,18,29,30]$.

Let $R$ is a prime ring with center $Z(R), Q$ is the Martindale quotient ring of $R$ and $U$ is the Utumi quotient ring of $R$. The center of $U$, denoted by $C$, is called the extended centroid of $R$. For any $x, y \in R$, the symbol $[x, y]$ and $x \circ y$ stands for the commutator $x y-y x$ and anti-commutator $x y+y x$, respectively. Given $x, y \in R$, we set $x \circ_{0} y=x, x \circ_{1} y=x \circ y=x y+y x$, and inductively $x \circ_{m} y=\left(x \circ_{m-1} y\right) \circ y$ for $m>1$.

Recall that a ring $R$ is prime if $x R y=\{0\}$ implies either $x=0$ or $y=0$, and $R$ is semiprime if $x R x=\{0\}$ implies $x=0$. An additive mapping $d: R \rightarrow R$ is called a derivation if $d(x y)=d(x) y+y d(x)$ holds, for all $x, y \in R$. In particular $d$ is an inner derivation induced by an element $q \in R$, if $d(x)=[q, x]$ holds, for all $x \in R$. If $R$ is a ring and $S \subseteq R$, a mapping $f: S \rightarrow R$ is called strong commutativity-preserving (scp) on $S$ if $[f(x), f(y)]=[x, y]$ for all $x, y \in S$. By Banach algebra we shall mean that complex normed algebra $\mathcal{A}$ whose underlying vector space is a Banach space. The Jacobson radical $\operatorname{rad}(\mathcal{A})$ of $\mathcal{A}$ be the intersection of all primitive ideals. If the Jacobson radical reduces to the zero element, $\mathcal{A}$ is called semisimple. In fact any Banach algebra $\mathcal{A}$ without a unity can be embedded into a unital Banach algebra $\mathcal{A}_{I}=\mathcal{A} \oplus \mathbb{C}$ as an ideal of codimension one. In particular, we may identify $\mathcal{A}$ with the ideal $\{(x, 0): x \in \mathcal{A}\}$ in $\mathcal{A}_{I}$ via the isometric isomorphism $x \rightarrow(x, 0)$.

Many results in literature indicate that the global structure of a ring $R$ is often tightly connected to the behaviour of additive mappings defined on $R$. Derivations with certain properties investigated in various papers (see $[6,13,28,31]$ and the references therein). Starting from these results, many authors studied generalized derivations in the context of prime and semiprime rings. By a generalized inner derivation on $R$, one usually means an additive mapping $F: R \rightarrow R$ if $F(x)=a x+x b$ for fixed $a, b \in R$. For such a mapping $F$, it is easy to see that $F(x y)=F(x) y+x[y, b]=F(x) y+x I_{b}(y)$, where $I_{b}$ is an inner derivation determined by $b$. This observation leads to the definition given in [8]: an additive mapping $F: R \rightarrow R$ is called generalized derivation associated with a derivation $d$ if $F(x y)=F(x) y+x d(y)$ for all $x, y \in R$. Familiar examples of generalized derivations are derivations and generalized inner derivations. Since the sum of two generalized derivations is a generalized derivation, every map of the form $F(x)=c x+d(x)$ is a generalized derivation, where $c$ is a fixed element of $R$ and $d$ is a derivation of $R$.

In [25], Lee extended the definition of a generalized derivation as follows: by a generalized derivation we mean an additive mapping $F: I \rightarrow U$ such that $F(x y)=$ $F(x) y+x d(y)$ holds, for all $x, y \in I$, where $I$ is a dense right ideal of $R$ and $d$ is a derivation from $I$ into $U$. Moreover, Lee also proved that every generalized derivation can be uniquely extended to a generalized derivation on $U$, and thus all generalized derivations of $R$ will be implicitly assumed to be defined on the derivation $F$ on dense 
right ideal of $R$ can be uniquely extended to $U$ and assumes the form $F(x)=a x+d(x)$ for some $a \in U$ and a derivation $d$ on $U$ (see [25, Theorem 3]).

Let us introduce the background of our investigation. The classical result of Singer and Wermer [33], states that any continuous derivation on a commutative Banach algebra has the range in the Jacobson radical of the algebra. Singer and Wermer also formulated the conjecture that the continuity assumption can be removed. In 1988 Thomas verified this conjecture [34]. It is clear that the same result of Singer and Wermer does not hold in non-commutative Banach algebras (because of inner derivations). However, this situation raise a very interesting question i.e., how to obtain the non-commutative version of the Singer-Wermer theorem. A first answer to this problem was obtained by Sinclair [32]. He proved that every continuous derivation of a Banach algebra leaves primitive ideals of the algebra invariant. Since then many authors obtained more information about derivations satisfying certain suitable conditions in Banach algebras.

The present paper is motivated by the previous results and we here continue this line of investigation by examining what happens if any one of the following conditions holds:

(i) $F(x) \circ_{m} F(y)=(x \circ y)^{n}$;

(ii) $F(x) \circ_{m} d(y)=d(x \circ y)^{n}$,

for all $x, y$ in some appropriate subset of $R$. We also examine the case where $R$ is a semiprime ring. Finally, we discuss the relationship between the structure of an algebra $\mathcal{A}$ and the behaviour of generalized derivations defined on $\mathcal{A}$. Then we apply our results on prime rings to the study of analogous conditions for continuous or spectrally bounded generalized derivations on Banach algebras.

\section{The Results in Prime Rings}

For the proof of our main results, we need the following facts, which might be of some independent interest.

Fact 2.1 ([11]). If $I$ is a two-sided ideal of $R$, then $R, I$ and $U$ satisfy the same generalized polynomial identities with coefficients in $U$.

Fact 2.2 ([4, Proposition 2.5.1]). Every derivation $d$ of $R$ can be uniquely extended to a derivation of $U$.

Fact 2.3 ([24]). If $I$ is a two-sided ideal of $R$, then $R, I$ and $U$ satisfy the same differential identities.

For a complete and detailed description of the theory of generalized polynomial identities involving derivations, we refer the reader to [4, Chapter 7]. We will make frequent use of the following result due to Kharchenko [22] (see also [24]).

Let $R$ be a prime ring, $d$ be a nonzero derivation of $R$ and $I$ be a nonzero two sided ideal of $R$. Let $f\left(x_{1}, \cdots x_{n}, d\left(x_{1}\right), \cdots d\left(x_{n}\right)\right)$ be a differential identity in $I$, that is

$$
f\left(r_{1}, \cdots r_{n}, d\left(r_{1}\right), \cdots, d\left(r_{n}\right)\right)=0, \quad \text { for all } r_{1}, \cdots, r_{n} \in I .
$$


One of the following holds:

(a) either $d$ is an inner derivation in $Q$, the Martindale quotient ring of $R$, in the sense that there exists $q \in Q$ such that $d=\operatorname{ad}(q)$ and $d(x)=\operatorname{ad}(q)(x)=[q, x]$, for all $x \in R$, and $I$ satisfies the generalized polynomial identity

$$
f\left(r_{1}, \cdots, r_{n},\left[q, r_{1}\right], \cdots,\left[q, r_{n}\right]\right)=0 ;
$$

(b) or, $I$ satisfies the generalized polynomial identity

$$
f\left(x_{1}, \cdots, x_{n}, y_{1}, \cdots, y_{n}\right)=0 .
$$

The following Lemma is very crucial for developing the proof of our main results.

Lemma 2.1. Let $R$ be a prime ring, $I$ be a nonzero ideal of $R$ and $n$ be a fixed positive integer. If $R$ admits a derivation $d$ such that $d(x \circ y)^{n}=0$ for all $x, y \in I$, then either $d=0$ or $R$ is commutative.

Proof. By the given hypothesis, we can write

$$
(d(x) y+x d(y)+d(y) x+y d(x))^{n}=0, \quad \text { for all } x, y \in I .
$$

In the light of Kharchenko's theorem [22], we split the proof into two cases.

Let $d$ be $Q$-outer derivation, then $I$ satisfies the polynomial identity $(s y+x t+t x$ $+y s)^{n}=0$, for all $x, y, s, t \in I$. In particular for $y=0, I$ satisfy the polynomial identity $(x t+t x)^{n}=0$ for all $x, t \in I$. If $\operatorname{char}(R) \neq 2$, then $\left(2 x^{2}\right)^{n}=0$ for all $x \in I$. This is a contradiction by $\mathrm{Xu}$ [35]. If $\operatorname{char}(R)=2$, then $(x y+y x)^{n}=0=[x, y]^{n}$ for all $s, y \in I$. Thus by Herstein [16, Theorem 2], we have $I \subseteq Z(R)$, and so $R$ is commutative by Mayne [27].

Let $d$ be an inner derivation induced by an element $q \in Q$ that is, $d(x)=[q, x]$ for all $x \in R$. It follows that, for any $x, y \in I$,

$$
([q, x] y+x[q, y]+[q, y] x+y[q, x])^{n}=0 .
$$

By Chuang [11, Theorem 2], $I$ and $Q$ satisfy same generalized polynomial identities (GPIs), thus (2.1) is satisfied by $Q$. In case center $C$ of $Q$ is infinite, then (2.1) is also satisfied by $Q \otimes_{C} \bar{C}$, where $\bar{C}$ is algebraic closure of $C$. Since both $Q$ and $Q \otimes_{C} \bar{C}$ are prime and centrally closed [14, Theorem 2.5 and Theorem 3.5], we may replace $R$ by $Q$ or $Q \otimes_{C} \bar{C}$ according as $C$ is finite or infinite. Thus we may assume that $R$ is centrally closed over $C$ (i.e., $R C=R$ ) which is either finite or algebraically closed and hence $R$ satisfy (2.1). Thus by Martindale [19, Theorem 3], $R C$ (and so $R$ ) is a primitive ring having nonzero socle $H$ with $\mathcal{D}$ as the associated division ring. It follows by Jacobson's theorem [20, p.75], $R$ is isomorphic to a dense ring of linear transformations of some vector space $\mathcal{V}$ over $\mathcal{D}$ and $H$ consists of the finite rank linear transformations in $R$. If $\mathcal{V}$ is a finite dimensional over $\mathcal{D}$. Then the density of $R$ on $\mathcal{V}$ implies that $R \cong \mathcal{M}_{k}(\mathcal{D})$, where $k=\operatorname{dim}_{\mathcal{D}} \mathcal{V}$. Suppose that $\operatorname{dim}_{\mathcal{D}} \mathcal{V} \geq 3$. First of all, we want to show that $v$ and $q v$ are linearly $\mathcal{D}$-dependent for all $v \in \mathcal{V}$. If $q v=0$, then $\{v, q v\}$ is $\mathcal{D}$-dependent. Suppose that $v \neq 0$. If $\{v, q v\}$ is $\mathcal{D}$-independent, as 
$\operatorname{dim}_{\mathcal{D}} \mathcal{V} \geq 3$, then there exists $w \in \mathcal{V}$ such that $\{v, q v, w\}$ are also $\mathcal{D}$-independent. By the density of $R$, there exist $x, y \in R$ such that

$$
\begin{aligned}
& x v=0, \quad x q v=0, \quad x w=v, \\
& y v=0, \quad y q v=w, \quad y w=0 .
\end{aligned}
$$

The above equations imply that $0=([q, x] y+x[q, y]+[q, y] x+y[q, x])^{n} v=(-1)^{n} v \neq 0$, a contradiction. So, we conclude that $\{v, q v\}$ is linearly $\mathcal{D}$-dependent for all $v \in \mathcal{V}$. We show here that there exists $\alpha \in \mathcal{D}$ such that $q v=v \alpha$, for any $v \in \mathcal{V}$. Note that the arguments in [10], are still valid in the present situation. For the sake of completeness and clearness we prefer to present it. In fact, we choose $v, w \in \mathcal{V}$ linearly independent. Since $\operatorname{dim}_{\mathcal{D}} \mathcal{V} \geq 3$, there exists $u \in \mathcal{V}$ such that $v, w, u$ are linearly independent. As already mention above, there exist $\alpha_{v}, \alpha_{w}, \alpha_{u} \in \mathcal{D}$ such that

$$
q v=v \alpha_{v}, \quad q w=w \alpha_{w}, \quad q u=u \alpha_{u}, \quad \text { that is } q(v+w+u)=v \alpha_{v}+w \alpha_{w}+u \alpha_{u} .
$$

Moreover $q(v+w+u)=(v+w+u) \alpha_{v+w+u}$, for a suitable $\alpha_{v+w+u} \in \mathcal{D}$. Then

$$
0=v\left(\alpha_{v+w+u}-\alpha_{v}\right)+w\left(\alpha_{v+w+u}-\alpha_{w}\right)+u\left(\alpha_{v+w+u}-\alpha_{u}\right),
$$

and, because $v, w, u$ are linearly independent, $\alpha_{u}=\alpha_{w}=\alpha_{v}=\alpha_{v+w+u}$, that is, $\alpha$ does not depend on the choice of $v$. So there exists $\alpha \in \mathcal{D}$ such that $q v=\alpha v$ for all $v \in \mathcal{V}$. Now for $r \in R, v \in \mathcal{V}$. Since $q v=v \alpha$ we have $[q, r] v=(q r) v-(r q) v=$ $q(r v)-r(q v)=(r v) \alpha-r(v \alpha)=0$, that is $[q, R] \mathcal{V}=0$. Since $\mathcal{V}$ is a left faithful irreducible $R$-module, hence $[q, R]=0$, i.e., $q \in Z(R)$ and so $d=0$, a contradiction.

Suppose now that $\operatorname{dim}_{\mathcal{D}} \mathcal{V} \leq 2$. In this case $R$ is a simple GPI-ring with 1 , and so it is a central simple algebra finite dimensional over its center. By Lanski [23, Lemma 2], it follows that there exists a suitable field $\mathbb{F}$ such that $R \subseteq \mathcal{M}_{k}(\mathbb{F})$, the ring of all $k \times k$ matrices over $\mathbb{F}$, and moreover, $\mathcal{M}_{k}(\mathbb{F})$ satisfies the same GPI as $R$. Assume $k \geq 3$, then by the same argument as above we can get a contradiction. Obviously if $k=1$, then $R$ is commutative. Thus we may assume that $k=2$, i.e., $R \subseteq \mathcal{M}_{2}(\mathbb{F})$, where $\mathcal{M}_{2}(\mathbb{F})$ satisfies $([q, x] y+x[q, y]+[q, y] x+y[q, x])^{n}=0$. Denote $e_{i j}$ the usual unit matrix with 1 in $(i, j)$-entry and zero elsewhere. Let $[x, y]=\left[e_{12}, e_{22}\right]=e_{12}$. then it is easy to see that $\left(q e_{12}-e_{12} q\right)^{n}=0$, left multiplying by $e_{12}$, one can get $e_{12}\left(q e_{12}\right)^{n}=0$. By setting

It easily follows that

$$
q=\left(\begin{array}{ll}
q_{11} & q_{12} \\
q_{21} & q_{22}
\end{array}\right)
$$

$$
\left(\begin{array}{cc}
0 & q_{21}^{n} \\
0 & 0
\end{array}\right)=0
$$

implies that $q_{12}=0$, on the other hand we can get $q_{21}=0$. Thus in all we see that that $q$ is a diagonal matrix in $\mathcal{M}_{2}(\mathbb{F})$. Let $\chi \in \operatorname{Aut}\left(\mathcal{M}_{2}(\mathbb{F})\right)$. Since

$$
([\chi(q), \chi(x)] \chi(y)+\chi(x)[\chi(q), \chi(y)]+[\chi(q), \chi(y)] \chi(x)+\chi(y)[\chi(q), \chi(x)])^{n}=0
$$

So, $\chi(q)$ must be diagonal matrix in $\mathcal{M}_{2}(\mathbb{F})$. In particular, let $\chi(x)=\left(1-e_{i j}\right) x\left(1+e_{i j}\right)$ for $i \neq j$. Then $\chi(q)=q+\left(q_{i i}-q_{j j}\right) e_{i j}$, that is $q_{i i}=q_{j j}$ for $i \neq j$. This implies 
that $q$ is central in $\mathcal{M}_{2}(\mathbb{F})$, which leads to $d=0$, a contradiction. This completes the proof.

Theorem 2.1. Let $R$ be a prime ring with characteristic different from 2 , $I$ be a nonzero ideal of $R$ and $m, n$ be the fixed positive integers. If $R$ admits a generalized derivation $F$ associated with a derivation d such that $F(x) \circ_{m} F(y)=(x \circ y)^{n}$ for all $x, y \in I$, then either $R$ is commutative or $d=0$ and there exist $a \in C$ such that $F(x)=$ ax for $x \in R$.

Proof. On contrary suppose that neither $R$ is commutative nor $d=0$. Since $R$ is a prime ring and $F$ is generalized derivation of $R$, by [25, Theorem 3], we can write $F(x)=a x+d(x)$ for some $a \in U$ and a derivation $d$ on $U$. Moreover, we have given that $F(x) \circ_{m} F(y)=(x \circ y)^{n}$, which can be rewritten as

$$
a x+d(x) \circ_{m} a y+d(y)=(x \circ y)^{n}, \quad \text { for all } x, y \in I .
$$

Equivalently we have

$$
a x \circ_{m} a y+d(x) \circ_{m} a y+a x \circ_{m} d(y)+d(x) \circ_{m} d(y)=(x \circ y)^{n}, \quad \text { for all } x, y \in I .
$$

Let $d$ be $Q$-outer derivation, then $I$ satisfies the polynomial identity

$$
a x \circ_{m} a y+s \circ_{m} a y+a x \circ_{m} t+s \circ_{m} t=(x \circ y)^{n}, \quad \text { for all } x, y, s, t \in I .
$$

In particular for $x=y=0, I$ satisfy the polynomial identity $s \circ_{m} t=0$ for all $s, t \in I$. By Chuang [11, Theorem 2], this polynomial identity is also satisfied by $Q$ and hence $R$ as well. Note that this is a polynomial identity and hence there exist a field $\mathbb{F}$ such that $R \subseteq \mathcal{M}_{k}(\mathbb{F})$, the ring of $k \times k$ matrices over a field $\mathbb{F}$, where $k \geq 1$. Moreover, $R$ and $\mathcal{M}_{k}(\mathbb{F})$ satisfy the same polynomial identity [23, Lemma 1], that is, $s \circ_{m} t=0$, for all $s, t \in \mathcal{M}_{k}(\mathbb{F})$. Denote $e_{i j}$ the usual matrix unit with 1 in $(i, j)$-entry and zero elsewhere. By choosing $s=e_{12}, t=e_{11}$, we see that $s \circ_{m} t=e_{12} \neq 0$, a contradiction.

Let $d$ be an inner derivation induced by an element $q \in Q$ that is, $d(x)=[q, x]$ for all $x \in R$. It follows that, for any $x, y \in I$,

$$
a x \circ_{m} a y+[q, x] \circ_{m} a y+a x \circ_{m}[q, y]+[q, x] \circ_{m}[q, y]=(x \circ y)^{n} .
$$

By Chuang [11, Theorem 2], $I$ and $Q$ satisfy same generalized polynomial identities (GPIs), thus (2.2) is satisfied by $Q$. In case center $C$ of $Q$ is infinite, then (2.2) is also satisfied by $Q \otimes_{C} \bar{C}$, where $\bar{C}$ is algebraic closure of $C$. Since both $Q$ and $Q \otimes_{C} \bar{C}$ are prime and centrally closed [14, Theorem 2.5 and Theorem 3.5], we may replace $R$ by $Q$ or $Q \otimes_{C} \bar{C}$ according as $C$ is finite or infinite. Thus we may assume that $R$ is centrally closed over $C$ (i.e., $R C=R$ ) which is either finite or algebraically closed and hence $R$ satisfy (2.2). Thus by Martindale [19, Theorem 3], $R C$ (and so $R$ ) is a primitive ring having nonzero socle $H$ with $\mathcal{D}$ as the associated division ring. It follows by Jacobson's theorem [20, p.75], $R$ is isomorphic to a dense ring of linear transformations of some vector space $\mathcal{V}$ over $\mathcal{D}$ and $H$ consists of the finite rank linear transformations in $R$. If $\mathcal{V}$ is a finite dimensional over $\mathcal{D}$. Then the density of $R$ on $\mathcal{V}$ 
implies that $R \cong \mathcal{M}_{k}(\mathcal{D})$, where $k=\operatorname{dim}_{\mathcal{D}} \mathcal{V}$. If $\operatorname{dim}_{\mathcal{D}} \mathcal{V}=1$, then $R$ is commutative, which contradicts our hypothesis. Suppose that $\operatorname{dim}_{\mathcal{D}} \mathcal{V} \geq 2$. First of all, we want to show that $v$ and $q v$ are linearly $\mathcal{D}$-dependent for all $v \in \mathcal{V}$. If $v=0$, then $\{v, q v\}$ is $\mathcal{D}$-dependent. Suppose that $v \neq 0$. If $\{v, q v\}$ is $\mathcal{D}$-independent, as $\operatorname{dim}_{\mathcal{D}} \mathcal{V} \geq 2$, by the density of $R$, there exist $x, y \in R$ such that

$$
x v=0, \quad x q v=-v, \quad y v=0, \quad y q v=v .
$$

The above equations imply that

$$
\begin{aligned}
0 & =\left(a x \circ_{m} a y+[q, x] \circ_{m} a y+a x \circ_{m}[q, y]+[q, x] \circ_{m}[q, y]-(x \circ y)^{n}\right) v \\
& =(-1)^{m} 2^{m} v, \quad \text { a contradiction. }
\end{aligned}
$$

So we conclude that $\{v, q v\}$ are linearly $\mathcal{D}$-dependent for all $v \in \mathcal{V}$. We show here that there exists $\beta \in \mathcal{D}$ such that $q v=v \beta$, for any $v \in \mathcal{V}$. Note that the arguments in [10], are still valid in the present situation. For the sake of completeness and clearness we prefer to present it. In fact, choose $v, w \in \mathcal{V}$ linearly independent. Since $\operatorname{dim}_{\mathcal{D}} \mathcal{V} \geq 2$, there exist $\beta_{v}, \beta_{w}, \beta_{v+w} \in \mathcal{D}$ such that

$$
q v=v \beta_{v}, \quad q w=w \beta_{w}, \quad q(v+w)=(v+w) \beta_{v+w} .
$$

Moreover

$$
v \beta_{v}+w \beta=(v+w) \beta_{v+w} .
$$

Hence

$$
v\left(\beta_{v}-\beta_{v+w}\right)+w\left(\beta_{w}-\beta_{v+w}\right)=0,
$$

and because $v, w$ are linearly $\mathcal{D}$-independent, we have $\beta_{v}=\beta_{w}=\beta_{v+w}$, that is, $\beta$ does not depend on the choice of $v$. So there exists $\beta \in \mathcal{D}$ such that $q v=\beta v$ for all $v \in \mathcal{V}$. Now for $r \in R, v \in \mathcal{V}$. Since $q v=v \beta$ we have $[q, r] v=(q r) v-(r q) v=$ $q(r v)-r(q v)=(r v) \beta-r(v \beta)=0$, that is $[q, R] \mathcal{V}=0$. Since $\mathcal{V}$ is a left faithful irreducible $R$-module, hence $[q, R]=0$, i.e., $q \in Z(R)$ and so $d=0$, a contradiction. This completes the proof of the theorem.

Theorem 2.2. Let $R$ be a prime ring with characteristic different from $2, I$ be a nonzero ideal of $R$ and $m, n$ be the fixed positive integers. If $R$ admits a generalized derivation $F$ associated with a derivation d such that $F(x) \circ_{m} d(y)=d(x \circ y)^{n}$ for all $x, y \in I$, then either $d=0$ or $R$ is commutative.

Proof. If $F=0$, then by Lemma 2.1, we are done, now consider the case when $F$ is a nonzero generalized derivation and $F(x) \circ_{m} d(y)=d(x \circ y)^{n}$ for all $x, y \in I$. By Lee [25, Theorem 3], $F(x)=a x+d(x)$ for some $a \in U$ and a derivation $d$ on $U$. This condition is a differential identity and therefore $I$ satisfy

$$
(a x+d(x)) \circ_{m} d(y)=d(x \circ y)^{n}, \quad \text { for all } x, y \in I .
$$

Which is rewritten as, for all $x, y \in I$

$$
\left(a x \circ_{m} d(y)\right)+\left(d(x) \circ_{m} d(y)\right)=((d(x) \circ y)+(x \circ d(y)))^{n} .
$$


In the light of Kharchenko's theorem [22], either $d=\operatorname{ad}(q)$ is the inner derivation induced by an element $a \in Q$, the Martindale quotient ring $R$, or $I$ satisfies the polynomial identity

$$
\left(a x \circ_{m} t\right)+\left(s \circ \circ_{m} t\right)=((s \circ y)+(x \circ t))^{n}, \quad \text { for all } x, y, s, t \in I .
$$

In the later case set $t=0$, to obtain the identity $(s y+y s)^{n}=0$ for all $s, y \in I$, and $R$ is commutative by Lemma 2.1. Assume now that $d=a d(q)$, that is $d(x)=[q, x]$. Then,

$$
\left(a x \circ_{m}[q, y]\right)+\left([q, x] \circ_{m}[q, y]\right)=(([q, x] \circ y)+(x \circ[q, y]))^{n},
$$

for all $x, y \in I$. As in the proof Lemma 2.1, we see that

(2.3) $\left(a x \circ_{m}[q, y]\right)+\left([q, x] \circ_{m}[q, y]\right)=(([q, x] \circ y)+(x \circ[q, y]))^{n}, \quad$ for all $x, y \in R$,

where $R$ is a primitive ring with $\mathcal{D}$ as the associated division ring. If $\mathcal{V}$ is finite dimensional over $\mathcal{D}$, then the density of $R$ implies that $R \cong \mathcal{M}_{k}(\mathcal{D})$, where $k=$ $\operatorname{dim}_{\mathcal{D}} \mathcal{V}$

Suppose that $\operatorname{dim}_{\mathcal{D}} \mathcal{V} \geq 2$, otherwise we are done. We want to show that $v$ and $q v$ are linearly $\mathcal{D}$-dependent for all $v \in \mathcal{V}$. If $q v=0$, then $\{v, q v\}$ is linearly $\mathcal{D}$-dependent. Suppose on contrary that $v$ and $q v$ are linearly $\mathcal{D}$-independent for some $v \in \mathcal{D}$.

If $q^{2} v \notin \operatorname{Span}_{\mathcal{D}}\{v, q v\}$, then $\left\{v, q v, q^{2} v\right\}$ is linearly $\mathcal{D}$-independent. By the density of $R$ there exist $x, y \in R$ such that

$$
x v=0, \quad x q v=q v, \quad x q^{2} v=v, \quad y v=0, \quad y q v=-v, \quad y q^{2} v=0 .
$$

The application of (2.3) implies that

$$
0=\left(a x \circ_{m}[q, y]+[q, x] \circ_{m}[q, y]-([q, x] \circ y+x \circ[q, y])^{n}\right) v=-v \neq 0,
$$

and we arrive at a contradiction.

If $q^{2} v \in \operatorname{Span}_{\mathcal{D}}\{v, q v\}$, then $q^{2} v=\alpha v+\gamma q v$ for some $\alpha, \gamma \in \mathcal{D}$. Since $v$ and $q v$ are linearly $\mathcal{D}$-independent, by the density of $R$, there exist $x, y \in R$ such that

$$
x v=0, \quad x q v=q v, \quad y v=0, \quad y q v=-v .
$$

It follows from the relation (2.3) that

$$
\begin{aligned}
0 & =\left(a x \circ_{m}[q, y]+[q, x] \circ_{m}[q, y]-([q, x] \circ y+x \circ[q, y])^{n}\right) v \\
& =-2^{m-1} \gamma v-v=2^{m-1} \lambda v \neq 0, \quad \text { for some } \lambda \in \mathcal{D},
\end{aligned}
$$

which is a contradiction. Thus we conclude that $v$ and $q v$ are linearly $\mathcal{D}$-dependent for all $v \in \mathcal{V}$. Thus we can write $q v=v \beta$ for all $v \in \mathcal{V}$ and a fixed $\beta \in \mathcal{D}$. Reasoning as in the proof of Lemma 2.1, we get required result.

The following examples show that the main results are not true in the case of arbitrary ring.

Example 2.1. Let $S$ be any ring. 
(i) Let

$$
R=\left\{\left(\begin{array}{ll}
a & b \\
0 & 0
\end{array}\right): a, b \in S\right\} \text { and } I=\left\{\left(\begin{array}{ll}
0 & a \\
0 & 0
\end{array}\right): a \in S\right\} .
$$

Then $R$ is a ring under usual operations and $I$ is a nonzero ideal of $R$. We define a map $F: R \rightarrow R$ by $F(x)=2 e_{11} x-x e_{11}$. Then it is easy to see that $F$ is a generalized derivation associated with a nonzero derivation $d(x)=e_{11} x-x e_{11}$. It is straightforward to check that for all positive integers $m, n, F$ satisfies the properties, $F(x) \circ_{m} F(y)=(x \circ y)^{n}$ and $F(x) \circ_{m} d(y)=d(x \circ y)^{n}$ for $x, y \in I$, however $R$ is not commutative.

(ii) Let $\mathbb{Z}$ be the ring of integers. Next, let

$$
R=\left\{\left(\begin{array}{ll}
a & b \\
0 & c
\end{array}\right): a, b, c \in \mathbb{Z}\right\} \text { and } I=\left\{\left(\begin{array}{ll}
0 & a \\
0 & 0
\end{array}\right): a \in \mathbb{Z}\right\} .
$$

Clearly, $R$ is a ring under the usual operations which is not prime. Define a map $F: R \rightarrow R$ by

$$
F\left(\begin{array}{ll}
a & b \\
0 & c
\end{array}\right)=\left(\begin{array}{cc}
a & b+a \\
0 & 0
\end{array}\right)
$$

It is easy to see that $F$ is a generalized derivation associated with a nonzero derivation

$$
d\left(\left(\begin{array}{ll}
a & b \\
0 & c
\end{array}\right)\right)=\left(\begin{array}{cc}
0 & a-c \\
0 & 0
\end{array}\right) .
$$

It is straightforward to check that $I$ is a nonzero ideal of $R$ and $F$ satisfies the properties, $F(x) \circ_{m} F(y)=(x \circ y)^{n}$ and $F(x) \circ_{m} d(y)=d(x \circ y)^{n}$ for $x, y \in I$, but $R$ is not commutative. Hence, the hypothesis of primeness is necessary.

\section{The Results in Semiprime Rings}

From now on, $R$ is a semiprime ring and $U$ is the left Utumi quotient ring of $R$. In order to prove the main result of this section we will make use of the following facts:

Fact 3.1 ([4, Proposition 2.5.1]). Any derivation of a semiprime ring $R$ can be uniquely extended to a derivation of its left Utumi quotient ring $U$, and so any derivation of $R$ can be defined on the whole $U$.

Fact 3.2 ([12, p.38]). If $R$ is semiprime then so its left Utumi quotient ring is also semiprime. The extended centroid $C$ of a semiprime ring coincides with the center of its left Utumi quotient ring.

Fact 3.3 ([12, p.42]). Let $B$ be the set of all the idempotents in $C$, the extended centroid of $R$. Suppose that $R$ is orthogonally complete $B$-algebra. For any maximal ideal $P$ of $B, P R$ forms a minimal prime ideal of $R$, which is invariant under any nonzero derivation of $R$.

We will prove the following. 
Theorem 3.1. Let $R$ be a semiprime ring, $U$ be the left Utumi quotient ring of $R$ and $m, n$ be the fixed positive integers. If $R$ admits a generalized derivation $F$ associated with a derivation $d$ such that $F(x) \circ_{m} F(y)=(x \circ y)^{n}$ for all $x, y \in R$, then there exists a central idempotent element e of $U$ such that on the direct sum decomposition $U=e U \oplus(1-e) U, d$ vanishes identically on $e U$ and the ring $(1-e) U$ is commutative.

Proof. Since $R$ is semiprime and $F$ is a generalized derivation of $R$, then by Lee [25, Theorem 3], $F(x)=a x+d(x)$ for some $a \in U$ and a derivation $d$ on $U$. We have given that

$$
a x \circ_{m} a y+d(x) \circ_{m} a y+a x \circ_{m} d(y)+d(x) \circ_{m} d(y)=(x \circ y)^{n},
$$

for all $x, y \in R$. By Fact 3.2, $Z(U)=C$, the extended centroid of $R$, and by Fact 3.1, the derivation $d$ can be uniquely extended on $U$. By Lee [24], $R$ and $U$ satisfy the same differential identities. Then

$$
a x \circ_{m} a y+d(x) \circ_{m} a y+a x \circ_{m} d(y)+d(x) \circ_{m} d(y)=(x \circ y)^{n},
$$

for all $x, y \in U$. Let $B$ be the complete Boolean algebra of idempotents in $C$ and $M$ be any maximal ideal of $B$. By Chuang [12, p.42], $U$ is orthogonally complete $B$-algebra, and by Fact 3.3, $M U$ is a prime ideal of $U$ which is $d$-invariant. Denote $\bar{U}=U / M U$ and $\bar{d}$ the derivation induced by $d$ on $\bar{U}$, i.e., $\bar{d}(\bar{u})=\overline{d(u)}$ for all $u \in U$. For any $\bar{x}, \bar{y} \in \bar{U}$,

$$
\overline{a x} \circ_{m} \overline{a y}+\bar{d}(\bar{x}) \circ_{m} \overline{a y}+\overline{a x} \circ_{m} \bar{d}(\bar{y})+\bar{d}(\bar{x}) \circ_{m} \bar{d}(\bar{y})=(\bar{x} \circ \bar{y})^{n} .
$$

It is obvious that $\bar{U}$ is prime. Therefore, by Theorem 2.1, we have either $\bar{U}$ is commutative or $\bar{d}=0$ in $\bar{U}$. That is, either $d(U) \subseteq M U$ or $[U, U] \subseteq M U$. In any case $d(U)[U, U] \subseteq M U$, for all $M$, where $M U$ runs over all minimal prime ideals of $U$. Therefore $d(U)[U, U] \subseteq \bigcap_{M} M U=0$, we obtain $d(U)[U, U]=0$.

By using the theory of orthogonal completion for semiprime rings [4, Chapter 3], it is clear that there exists a central idempotent element $e$ in $U$ such that on the direct sum decomposition $U=e U \oplus(1-e) U, d$ vanishes identically on $e U$ and the ring $(1-e) U$ is commutative. This completes the proof of the theorem.

Using arguments similar to those used in the proof of the Theorem 3.1, we may conclude with the following (we omit the proof brevity). We can prove the following.

Theorem 3.2. Let $R$ be a semiprime ring with characteristic different from $2, U$ be the left Utumi quotient ring of $R$ and $m, n$ be the fixed positive integers. If $R$ admits a generalized derivation $F$ associated with a derivation $d$ such that $F(x) \circ_{m} d(y)=$ $d(x \circ y)^{n}$ for all $x, y \in R$, then there exists a central idempotent element e of $U$ such that on the direct sum decomposition $U=e U \oplus(1-e) U$, d vanishes identically on $e U$ and the ring $(1-e) U$ is commutative. 


\section{Applications on Non-Commutative Banach Algebras}

This section deals with application of our main results. In this section we obtain some results on non-commutative Banach algebras by using the preceding algebraic results. We apply the purely algebraic results which is obtained in section 2 and obtain the conditions that every continuous derivation on a Banach algebra maps into the radical. The proofs of the results rely on a Sinclair's theorem [32] which states that every continuous derivation $d$ of a Banach algebra $\mathcal{A}$ leaves the primitive ideals of $\mathcal{A}$ invariant. As we have mentioned before, Thomas [34], has generalized the Singer-Wermer theorem by proving that any derivation on a commutative Banach algebra maps the algebra into its radical.

This result leads us an important question whether the theorem can be proved without using any commutativity assumption. On this note, there are many papers $[19,26,32]$ which shows that the theorem holds without any commutativity assumption. We also acquire that every derivation maps into its radical with some property without any commutativity assumption.

Our first result in this section concerns about the continuous generalized derivations on Banach algebra.

Lemma 4.1. Let $\mathcal{A}$ be a non-commutative Banach algebra with Jacobson radical $\operatorname{rad}(\mathcal{A})$ and $n$ be a fixed positive integer. Suppose that there exist a continuous derivation $d: \mathcal{A} \rightarrow \mathcal{A}$ such that $d(x \circ y)^{n} \in \operatorname{rad}(\mathcal{A})$ for all $x, y \in \mathcal{A}$, then $d$ maps into the radical of $\mathcal{A}$.

Proof. By a result of Sinclair [32, Lemma 3.2], every continuous derivation $d$ on a Banach algebra $\mathcal{A}$ leaves the primitive ideals of $\mathcal{A}$ invariant. Since the Jacobson radical is the intersection of all primitive ideals, we have $d(\operatorname{rad}(\mathcal{A})) \subseteq \operatorname{rad}(\mathcal{A})$, which means that there is no loss of generality in assuming that $\mathcal{A}$ is semisimple. Since $d$ leaves all primitive ideals invariant, one can introduce for any primitive ideal $P \subseteq \mathcal{A}$, a nonzero derivation

$$
\begin{aligned}
& d_{P}: \mathcal{A} / P \rightarrow \mathcal{A} / P, \quad \text { where } \mathcal{A} / P=\overline{\mathcal{A}} \text { is a factor Banach algebra, } \\
& d_{P}(r+P)=d(r)+P, \quad \text { for all } r \in \mathcal{A} .
\end{aligned}
$$

Note that every derivation on a semisimple Banach algebra is continuous [21, Remark 4.3]. First, in case $\mathcal{A} / P$ is commutative, combining this result with the Singer-Wermer theorem gives $d_{P}=0$, as $\mathcal{A} / P$ is semisimple. We intend to show that $d_{P}=0$ in case when $\mathcal{A} / P$ is non-commutative. Since the assumption of the theorem gives $d(x \circ y)^{n} \in \operatorname{rad}(\mathcal{A})$ for all $x, y \in \mathcal{A}$, it follows that $d_{P}((x \circ y)+P)^{n}=0$ for all $x, y \in \mathcal{A}$. By Lemma $2.1, d_{P}=0$ on $\mathcal{A} / P$. Consequently $d(\mathcal{A}) \subseteq \mathcal{A}$ for any primitive ideal $P$. Since $\operatorname{rad}(\mathcal{A})$ is the intersection of all primitive ideal in $\mathcal{A}, d(\mathcal{A}) \subseteq \operatorname{rad}(\mathcal{A})$.

Corollary 4.1. Let $\mathcal{A}$ be a non-commutative semisimple Banach algebra with Jacobson radical $\operatorname{rad}(\mathcal{A})$ and $n$ be a fixed positive integer. Let $d: \mathcal{A} \rightarrow \mathcal{A}$ be a continuous derivation. If $d(x \circ y)^{n} \in \operatorname{rad}(\mathcal{A})$ for all $x, y \in \mathcal{A}$, then $d=0$. 
Proof. It is well-known that any derivation on semisimple Banach algebra is continuous [21]. By Lemma 4.1, $d(\mathcal{A}) \subseteq \operatorname{rad}(\mathcal{A})$. Since $\operatorname{rad}(\mathcal{A})=0, d(\mathcal{A})=0$.

Theorem 4.1. Let $\mathcal{A}$ be a non-commutative Banach algebra with Jacobson radical $\operatorname{rad}(\mathcal{A})$ and $m, n$ be the fixed positive integers. Let $F(x)=a x+d(x)$ be a continuous generalized derivation of $\mathcal{A}$ for some element $a \in \mathcal{A}$ and some derivation $d$ on $\mathcal{A}$. If $F(x) \circ_{m} F(y)-(x \circ y)^{n} \in \operatorname{rad}(\mathcal{A})$ for all $x, y \in \mathcal{A}$, then $d(\mathcal{A}) \subseteq \operatorname{rad}(\mathcal{A})$.

Proof. In view of Lemma 4.1, we only discussed the case when $F$ is a nonzero generalized derivation. Under the assumption that $F$ is continuous, and since it is well known that the left multiplication map is also continuous, we have that the derivation $d$ is continuous. In [32], Sinclair proved that any continuous derivation of a Banach algebra leaves the primitive ideals invariant. Therefore, for any primitive ideal $P$ of $\mathcal{A}$, it is follows that $F(P) \subseteq a P+d(P) \subseteq P$. It means that the continuous generalized derivation $F$ leaves the primitive ideals invariant. Denote $\mathcal{A} / P=\overline{\mathcal{A}}$ for any primitive ideals $P$. Hence we may introduced a nonzero generalized derivation

$$
F_{P}: \overline{\mathcal{A}} \rightarrow \overline{\mathcal{A}}
$$

such that

$$
F_{P}(\bar{r})=F_{P}(r+P) \subseteq F(r)+P=a r+d(r)+P,
$$

for all $r \in \mathcal{A}$ and $\bar{r}=r+P$, where $\mathcal{A} / P=\overline{\mathcal{A}}$ is a factor Banach algebra. Note that every derivation on a semisimple Banach algebra is continuous [21, Remark 4.3]. First, in case $\mathcal{A} / P$ is commutative, combining this result with the Singer-Wermer theorem gives $F_{P}=0$ since $\mathcal{A} / P$ is semisimple. We intend to show that $F_{P}=0$ in case when $\mathcal{A} / P$ is non-commutative. Moreover, by $F(x) \circ_{m} F(y)-(x \circ y)^{n} \in \operatorname{rad}(\mathcal{A})$ for all $x, y \in \mathcal{A}$, it follows that $\bar{F}(\bar{x}) \circ_{m} \bar{F}(\bar{y})-(\bar{x} \circ \bar{y})^{n}=\overline{0}$ for all $\bar{x}, \bar{y} \in \overline{\mathcal{A}}$. Since $\overline{\mathcal{A}}$ is primitive, a fortiori it is prime. Thus by Theorem 2.1, it is immediate that either $\overline{\mathcal{A}}$ is commutative or $d=\overline{0}$; that is, $[\mathcal{A}, \mathcal{A}] \subseteq P$ or $d(\mathcal{A}) \subseteq P$.

Now let $P$ be a primitive ideal such that $\overline{\mathcal{A}}$ is commutative. Singer and Wermer [33] proved that any continuous linear derivation on a commutative Banach algebra maps the algebra into its radical. Moreover, by a result of Johnson and Sinclair [21], any derivation on a semisimple Banach algebra is continuous. Hence there are no nonzero linear continuous derivations on commutative semisimple Banach algebras. Therefore $d=\overline{0}$ in $\overline{\mathcal{A}}$. Hence in any case we get $d(\mathcal{A}) \subseteq P$ for all primitive ideal $P$ of $\mathcal{A}$. Since radical $\operatorname{rad}(\mathcal{A})$ of $\mathcal{A}$ is the intersection of all primitive ideals, we get the required conclusion.

In view of the Theorem 4.1, we may prove the following corollary in the special case when $\mathcal{A}$ is a semisimple Banach algebra.

Corollary 4.2. Let $\mathcal{A}$ be a non-commutative semisimple Banach algebra with Jacobson radical $\operatorname{rad}(\mathcal{A})$ and $m, n$ be the fixed positive integers. Let $F(x)=a x+d(x)$ be a continuous generalized derivation of $\mathcal{A}$ for some element $a \in \mathcal{A}$ and some derivation $d$ on $\mathcal{A}$. If $F(x) \circ_{m} F(y)-(x \circ y)^{n} \in \operatorname{rad}(\mathcal{A})$ for all $x, y \in \mathcal{A}$, then $d(\mathcal{A})=0$. 
Proof. In view of Corollary 4.1, we may prove the result in the same way as Theorem 4.1, we omit the proof for brevity. Just let us remark that at the beginning of the proof one has to use the fact that the derivation $d$ is continuous in a semisimple Banach algebra (see [32]). Moreover, any left multiplication map is continuous, also $F$ is continuous. Finally, we use the fact that $\operatorname{rad}(\mathcal{A})=0$, since $\mathcal{A}$ is semisimple.

Using arguments similar to those used in the proof of the Theorem 4.1 and Lemma 4.1, we may conclude with the following (we omit the proof brevity). We can prove the following.

Theorem 4.2. Let $\mathcal{A}$ be a non-commutative Banach algebra with Jacobson radical $\operatorname{rad}(\mathcal{A})$ and $m, n$ be the fixed positive integers. Let $F(x)=a x+d(x)$ be a continuous generalized derivation of $\mathcal{A}$ for some element $a \in \mathcal{A}$ and some derivation d on $\mathcal{A}$. If $F(x) \circ_{m} d(y)-d(x \circ y)^{n} \in \operatorname{rad}(\mathcal{A})$ for all $x, y \in \mathcal{A}$, then $d(\mathcal{A}) \subseteq \operatorname{rad}(\mathcal{A})$.

\section{Spectrally Boundedness of Generalized Derivations}

Before beginning the proof of the main theorem, for the sake of completeness, we prefer to recall some basic notations, definitions and some easy consequences of the result of Brešar and Mathieu [9], about the spectrally bounded generalized derivation on Banach algebra. In [9, Theorem 2.8], Brešar and Mathieu obtained a necessary and sufficient condition for a generalized derivation to be spectrally bounded on a unital Banach algebra. Here $\delta=L_{a}+d$ will denote spectrally bounded generalized derivation.

A linear mapping $\delta$ on $\mathcal{A}$ is said to be a generalized derivation if

$$
\delta(x y z)=\delta(x y) z-x \delta(y)+x \delta(y z), \quad \text { for all } x, y, z \in \mathcal{A} .
$$

In the application such operators correspond to irreversible dynamics while derivations generate reversible ones. Put $a=\delta(1)$. Using (5.1), it is easily computed that $d(x)=\delta(x)-a x$, for all $x \in \mathcal{A}$ defines a derivation on $\mathcal{A}$. Hence, every generalized derivation $\delta$ is of the form $\delta=L_{a}+d$ with $a=\delta(1)$ and $d$ a derivation, and every generalized inner derivation is given by $\delta_{a}+d_{b}=L_{a-b}+\mathcal{R}_{b}$ (here, $L_{a}$ and $\mathcal{R}_{b}$ denote the left and right multiplication by $a$ and $b$, respectively). A spectrally bounded generalized derivation need not map into radical, but if it is inner, both its constituents $L_{a}$ and $d_{b}$ have to be spectrally bounded.

The last result of this paper has the same behaviour as the Theorem 4.1. We now turn our attention to the spectrally bounded generalized derivations. In order to prove our main theorem of this section we will use some results concerning spectrally bounded derivations and generalized derivations, more precisely, we need the following:

Lemma 5.1 ([9, Theorem 2.5]). Every spectrally bounded derivation on a unital Banach algebra maps the algebra into the radical.

Lemma 5.2 ([9, Lemma 2.7]). Every spectrally bounded generalized derivation leaves each primitive ideal invariant. 
Lemma 5.3 ([9, Theorem 2.8]). Let $\delta=L_{a}+d$ be a generalized derivation on a unital Banach algebra $\mathcal{A}$, where $L_{a}$ is the left multiplication (by the element a) map and $d$ some derivation of $\mathcal{A}$. Then $\delta$ is spectrally bounded if and only if both $L_{a}$ and $d$ are spectrally bounded.

Theorem 5.1. Let $\mathcal{A}$ be a non-commutative Banach algebra with Jacobson radical $\operatorname{rad}(\mathcal{A})$ and $m, n$ be the fixed positive integers. Let $\delta=L_{a}+d$ be a spectrally bounded generalized derivation of $\mathcal{A}$, where $L_{a}$ denote the left multiplication by some element $a \in \mathcal{A}$ and $d$ is a derivation of $\mathcal{A}$. If $\delta(x) \circ_{m} \delta(y)-(x \circ y)^{n} \in \operatorname{rad}(\mathcal{A})$ for all $x, y \in \mathcal{A}$, then $d(\mathcal{A}) \subseteq \operatorname{rad}(\mathcal{A})$.

Proof. Since $\delta$ is spectrally bounded, by Lemma $5.3, L_{a}$ and $d$ are spectrally bounded. Combining this with Lemma 5.2 we have that $d(\mathcal{A}) \subseteq \operatorname{rad}(\mathcal{A})$. In [32], Sinclair proved that any continuous derivation of a Banach algebra leaves the primitive ideals invariant. Hence, for any primitive ideal $P$ of $\mathcal{A}$, it is obvious that $\delta(P) \subseteq P$. It means that the continuous generalized derivation $\delta$ leaves the primitive ideals invariant. Thus we can define the generalized derivation $\delta_{P}: \overline{\mathcal{A}} \rightarrow \overline{\mathcal{A}}$ by $\delta_{P}(\bar{x})=\delta_{P}(x+P)=\delta_{P}(x)+P \subseteq$ $a x+d(x)+P \subseteq a x+P$ for all $\bar{x} \in \overline{\mathcal{A}}$, where $\mathcal{A} / P=\overline{\mathcal{A}}$ is a factor Banach algebra. Since $P$ is a primitive ideal, the factor algebra $\overline{\mathcal{A}}$ is primitive and so it is prime. The hypothesis $\delta(x) \circ_{m} \delta(y)-(x \circ y)^{n} \in \operatorname{rad}(\mathcal{A})$ yields that $\delta(\bar{x}) \circ_{m} \delta(\bar{y})-(\bar{x} \circ \bar{y})^{n}=\overline{0}$ for all $\bar{x}, \bar{y} \in \overline{\mathcal{A}}$. By Theorem 2.1, it is immediate that either $\overline{\mathcal{A}}$ is commutative or $d=\overline{0}$; that is, $[\mathcal{A}, \mathcal{A}] \subseteq P$ or $d(\mathcal{A}) \subseteq P$. Now we assume that $P$ is a primitive ideal such that $\overline{\mathcal{A}}$ is commutative. In [33], Singer and Werner proved that any continuous linear derivation on a commutative Banach algebra maps the algebra into the radical. Furthermore by a result of Jonhson and Sinclair [21], any linear derivation on semisimple Banach algebra is continuous. We know that there are no nonzero linear continuous derivations on commutative semisimple Banach algebras.

Therefore, $d=\overline{0}$ in $\overline{\mathcal{A}}$. Hence in any case we get $d(\mathcal{A}) \subseteq P$ for all primitive ideal $P$ of $\mathcal{A}$. Since radical $\operatorname{rad}(\mathcal{A})$ of $\mathcal{A}$ is the intersection of all primitive ideals, we get the required conclusion.

By using the same argument as in Theorem 5.1, we may conclude with the following (we omit the proof brevity).

Theorem 5.2. Let $\mathcal{A}$ be a non-commutative Banach algebra with Jacobson radical $\operatorname{rad}(\mathcal{A})$ and $m, n$ two fixed positive integers. Let $\delta=L_{a}+d$ be a spectrally bounded generalized derivation of $\mathcal{A}$, where $L_{a}$ denote the left multiplication by some element $a \in \mathcal{A}$ and $d$ is a derivation of $\mathcal{A}$. If $\delta(x) \circ_{m} d(y)-d(x \circ y)^{n} \in \operatorname{rad}(\mathcal{A})$ for all $x, y \in \mathcal{A}$, then $d(\mathcal{A}) \subseteq \operatorname{rad}(\mathcal{A})$.

Acknowledgements. The authors wishes to thank the referees for their valuable suggestions. 


\section{REFERENCES}

[1] S. Ali, M. Ashraf, M. S. Khan and J. Vukman, Commutativity of rings involving additive mappings, Quaest. Math. 37 (2014), 1-15.

[2] N. Argaç and H. G. Inceboz, Derivation of prime and semiprime rings, J. Korean Math. Soc. 46 (2009), 997-1005.

[3] M. Ashraf and N. Rehman, On commutativity of rings with derivations, Results Math. 42 (2002), $3-8$.

[4] K. I. Beidar, W. S. M. III and A. V. Mikhalev, Rings with Generalized Identities, Pure and Applied Mathematics, Marcel Dekker 196, New York, 1996.

[5] H. E. Bell and M. N. Daif, On commutativity and strong commutativity-preserving maps, Canad. Math. Bull. 37 (1994), 443-447.

[6] H. E. Bell and M. N. Daif, On derivations and commutativity in prime rings, Acta Math. Hung. 66 (1995), 337-343.

[7] H. E. Bell and N. Rehman, Generalized derivations with commutativity and anti-commutativity conditions, Math. J. Okayama Univ. 49 (2007), 139-147.

[8] M. Brešar, On the distance of the composition of two derivations to the generalized derivations, Glasgow Math. J. 33 (1991), 89-93.

[9] M. Brešar and M. Mathieu, Derivations mapping into the radical III, J. Funct. Anal. 133 (1995), 21-29.

[10] L. Carini and V. D. Filippis, Commuatators with power central values on a lie ideal, Pacific. J. Math. 193 (2000), 269-278.

[11] C. L. Chuang, GPIs having coefficients in Utumi quotient rings, Proc. Amer. Math. Soc. 103 (1988), 723-728.

[12] C. L. Chuang, Hypercentral derivations, J. Algebra 66 (1994), 34-71.

[13] M. N. Daif and H. E. Bell, Remarks on derivations on semiprime rings, Int. J. Math. Math. Sci. 15 (1992), 205-206.

[14] T. S. Erickson, W. S. M. III and J. M. Osborn, Prime nonassociative algebras, Pacific. J. Math. 60 (1975), 49-63.

[15] V. D. Filippis and S. Huang, Generalized derivations on semiprime rings, Bull. Korean Math. Soc. 48 (2011), 1253-1259.

[16] I. N. Herstein, Center-like elements in prime rings, J. Algebra 60 (1979), 567-574.

[17] S. Huang, Generalized derivation of prime rings, International Journal of Mathematics and Mathematical Sciences 2007 (2011), Article ID 85612.

[18] S. Huang, Generalized derivations of prime and semiprime rings, Taiwanese J. Math. 6 (2012), $771-776$.

[19] W. S. M. III, Prime rings satisfying a generalized polynomial identity, J. Algebra 12 (1969), $576-584$.

[20] N. Jacobson, Structure of Rings, Colloquium Publications 37, Amer. Math. Soc. VII, Provindence, RI, 1956.

[21] B. E. Johnson and A. M. Sinclair, Continuity of derivations and a problem of Kaplansky, Amer. J. Math. 90 (1968), 1067-1073.

[22] V. K. Kharchenko, Differential identities of prime rings, Algebra Logic 17 (1979), 155-168.

[23] C. Lanski, An Engel condition with derivation, Proc. Amer. Math. Soc. 118 (1993), 731-734.

[24] T. K. Lee, Semiprime rings with differential identities, Bull. Inst. Math. Acad. Sinica 20 (1992), $27-38$.

[25] T. K. Lee, Generalized derivations of left faithful rings, Comm. Algebra 27 (1998), 4057-4073.

[26] M. Mathieu and G. J. Murphy, Derivations mapping into the radical, Arch. Math. 57 (1991), 469-474.

[27] J. H. Mayne, Centralizing mappings of prime rings, Canad. Math. Bull. 27 (1984), 122-126.

[28] E. C. Posner, Derivations in prime rings, Proc. Amer. Math. Soc. 8 (1958), 1093-1100. 
[29] M. A. Raza and N. Rehman, On prime and semiprime rings with generalized derivations and non-commutative Banach algebras, Proceedings - Mathematical Sciences 126(3) (2016), 389-398.

[30] N. Rehman, M. A. Raza and T. Bano, On commutativity of rings with generalized derivations, J. Egyptian Math. Soc. 24 (2016), 151-155.

[31] N. Rehman, M. A. Raza and S. Huang, On generalized derivations in prime ring with skewcommutativity conditions, Rend. Circ. Math. Palermo (2) 64 (2015), 251-259.

[32] A. M. Sinclair, Continuous derivations on Banach algebras, Proc. Amer. Math. Soc. 20 (1969), 166-170.

[33] I. M. Singer and J. Wermer, Derivations on commutative normed algebras, Math. Ann. 129 (1955), 260-264.

[34] M. P. Thomas, The image of a derivation is contained in the radical, Ann. Math. 128 (1988), 435-460.

[35] X. W. Xu, The Power Values Properties of Generalized Derivations, Doctoral Thesis of Jilin University, Changchun, 2006.

${ }^{1}$ Department of Mathematics,

Aligarh Muslim University,

Aligarh-202002 IndiA

E-mail address: arifraza03@gmail.com

${ }^{1}$ Department of Mathematics,

Aligarh Muslim University,

AligarH-202002 IndiA

E-mail address: rehman100@gmail.com 